\title{
INVESTIGACIÓN EN POLÍTICAS Y SISTEMAS DE SALUD PARA LA GESTIÓN BASADA EN EVIDENCIAS
}

\author{
HEALTH RESEARCH POLICY AND SYSTEMS FOR MANAGEMENT BASED ON EVIDENCES
}

\author{
Aníbal Velásquez ${ }^{1, a}$
}

En la medida que los gerentes, autoridades y funcionarios del sector salud desarrollen una gestión en base a la mejor evidencia disponible, será más viable fortalecer el sistema de salud para una protección social más efectiva, equitativa, eficiente y de mejor calidad. El diseño de las políticas y programas requiere evidencias que justifiquen su formulación. Esta evidencia deberá describir la magnitud y severidad del problema, las necesidades de la población objetivo, identificar las áreas prioritarias, la situación de la oferta de servicios y la acción del Estado.

Las políticas y programas necesitan identificar intervenciones efectivas que tengan base científica, establecer metas y estrategias de expansión de coberturas de estas intervenciones, evaluar las restricciones que impiden que las intervenciones y servicios lleguen a los más vulnerables, analizar el costo de incrementar las coberturas de estas intervenciones, el presupuesto disponible y el presupuesto adicional para expandir las coberturas. También es necesario un análisis de viabilidad política, técnica, económica y social de las recomendaciones provenientes de las evaluaciones e investigaciones para que puedan implementarse en los servicios de salud.

La implementación de las políticas y los programas requiere información acerca de los usuarios, las coberturas de los servicios o beneficios, el cumplimiento de las actividades de los planes operativos y ejecución presupuestal, el seguimiento de los indicadores de desempeño, la identificación de barreras y facilitadores de las tareas y actividades, la percepción de los usuarios sobre la eficacia y calidad de los productos y servicios, y el seguimiento de los compromisos y recomendaciones que se hacen para hacer mejoras a los programas.

En este sentido la Investigación en Políticas y Sistemas de Salud debería ser la principal fuente de información porque estudia los problemas que tienen repercusiones en el desempeño del sistema de salud; desde el financiamiento, la gobernanza y las políticas hasta los problemas de estructuración, planificación, gestión, recursos humanos, prestación de servicios, y calidad de la atención en el sector público y privado ${ }^{(1)}$. Estas investigaciones pueden emplearse en varios momentos del ciclo de las políticas y analizan cualquier aspecto de la implementación de políticas, especialmente los factores que la afectan, los procesos de implementación en sí mismos y los resultados o productos finales de las políticas y programas ${ }^{(2,3)}$.

El monitoreo y la evaluación de las políticas, forma parte de estas investigaciones, y tienen como objetivo medir el progreso de una intervención de salud y determinar si está teniendo el impacto deseado ${ }^{(1)}$. Las evaluaciones de impacto y de resultados de las políticas y programas son importantes para decidir la continuidad de estas o, en todo caso, su rediseño.

Asimismo, se necesita desarrollar y probar soluciones para superar los obstáculos de la implementación e introducción de innovaciones en el sistema de salud o de promover su uso a gran escala y luego evaluar su sostenibilidad. Estas investigaciones deben ser rigurosas para determinar la eficacia, mediante estudios pilotos que ensayen variantes o mejoras que puedan implementarse para lograr intervenciones más efectivas, más simples o menos complejas y menos costosas ${ }^{(2)}$.

El uso de las evidencias por los tomadores de decisiones no es un proceso automático, es decir, el hecho de publicar no se traduce inmediatamente en el diseño o mejora de las políticas o del sistema de salud. Existen aspectos que

Pontificia Universidad Católica del Perú. Lima, Perú.

Médico epidemiólogo (ex Ministro de Salud del Perú)

Recibido: 26/09/2018 Aprobado: 26/09/2018 En línea: 28/09/2018

Citar como: Velásquez A. Investigación en políticas y sistemas de salud para la gestión basada en evidencias. Rev Peru Med Exp Salud Publica. 2018;35(3):3712. doi: $10.17843 /$ rpmesp.2018.353.3978 
favorecen el uso de las evidencias, así como barreras que la impiden ${ }^{(4)}$. Los científicos se ven a si mismos como racionales, con nuevas ideas y perciben a los tomadores de decisiones como personas con intereses particulares e indiferentes al conocimiento científico y a las nuevas ideas. Por otro lado, los tomadores de decisiones, se ven como responsables, orientados a la acción y pragmáticos, percibiendo a los científicos como ingenuos, con su propio lenguaje y sin compromiso con la implementación de políticas.

Si la evidencia generada es inoportuna o entra en conflicto con los intereses políticos, ideologías, la cultura institucional o las fuentes de información consideradas fidedignas, la probabilidad de uso de la misma se reduce. En consecuencia, es importante hacer una gestión de la evidencia y de las recomendaciones de las investigaciones para que estas puedan ser incorporadas a las decisiones en materia de planeamiento, diseño y operación. Esto implica una estrategia de relacionamiento y mejorar la comunicación entre los investigadores y políticos, a cargo de un personal especializado y dedicado a esta tarea.

Las recomendaciones requieren de una respuesta explícita por parte de las autoridades y decisores, la misma que puede adoptar compromisos y un plan de acción, especificando claramente las responsabilidades y metas. Luego de establecido un acuerdo para la implementación de las recomendaciones, es preciso realizar un seguimiento sistemático del avance de dicho proceso ${ }^{(5)}$.

En el Perú existen diversos ejemplos de uso de los resultados de la Investigación en Políticas y Sistemas de Salud. Se destaca el diseño e implementación de la política y programa de presupuesto por resultados para la reducción de la desnutrición crónica infantil (DCI), el cual ha tenido resultados reconocidos a nivel mundial, por haber reducido
15 puntos porcentuales la prevalencia de $\mathrm{DCl}$ en un periodo de ocho años.

También se reconoce el rol del Instituto Nacional de Salud en los estudios de resistencia de la malaria a la quimioterapia que permitieron modificar la norma técnica que contribuyó a reducir la prevalencia de este mal en el país, así como los aportes de la Unidad de Análisis y Generación de Evidencias en Salud Pública (UNAGESP) en el diseño de programas de presupuesto por resultados y en importantes decisiones del Ministerio de Salud.

Como editor invitado para el simposio dedicado a la Investigación en Políticas y Sistemas de Salud invité a destacados profesionales para publicar sus contribuciones. Este número publica tres artículos que describen experiencias sobre la implementación de políticas de atención en $\mathrm{VIH}$, antimaláricos, y el fortalecimiento del Sistema de Información de la Mortalidad en el Perú. Felicito a la Revista Peruana de Medicina Experimental y Salud Pública por esta iniciativa, y por su cooperación con la Academia Nacional de Medicina para la organización de un Simposio en el cuál se discutirán los resultados y recomendaciones de las investigaciones presentadas. De esta forma se pone en práctica la recomendación ${ }^{(4)}$ de que para incrementar la gestión basada en evidencias es necesario establecer lazos entre los investigadores, los políticos y los gerentes.

Por último, invocamos a los profesionales e investigadores del Perú, a reconocer la importancia de la Investigación en Políticas y Sistemas de Salud. Este tipo de investigación es completamente viable, requiere principalmente de dedicación para utilizar las bases de datos administrativas, que cada vez son de mejor calidad, analizar bases de datos de encuestas poblacionales y censos nacionales, y recursos adecuados para realizar investigaciones cualitativas, revisiones sistemáticas y documentales.

\section{REFERENCIAS BIBLIOGRÁFICAS}

1. Remme J. et al. Defining Research to Improve Health Systems, PLoS Med. 2010; 7(11): e1001000. doi: 10.1371/journal. pmed.1001000.

2. David H. Peters, Nhan T. Tran, Taghreed Adam. Investigación sobre la implementación de políticas de salud: Guía práctica. Alianza para la Investigación en Políticas y Sistemas de Salud, Organización Mundial de la Salud, 2013. http:// www.who.int/alliance-hpsr/resources/ Implementation_Research_SP.pdf

3. WHO. The Alliance for Health Policy and Systems Research. What is Health Policy and Systems Research (HPSR)? http://www.who.int/alliance-hpsr/ about/hpsr/en/

4. Innvaer S, Vist G, Trommald M, Oxman A. Health policy-makers' perceptions of their use of evidence: a systematic review,
Health Services Research Unit, National Institute of Public Health, Oslo, Norway; J Health Serv Res Policy. 2002; 7(4): 23944. doi: $10.1258 / 135581902320432778$

5. Velásquez A. ¿Cómo usar la evidencia para mejorar programas y políticas? en: BID, Banco Mundial, CEPLAN. (2015). Los sistemas de monitoreo y evaluación: hacia la mejora continua de la planificación. Pag. 98-101 estratégica y la gestión pública. 